\title{
Electrodynamics of electric power transmission and losses in devices of electric transport systems
}

\author{
Mykola Kostin ${ }^{1, *}$ \\ ${ }^{1}$ Dnipropetrovsk National University of Railway Transport named after Academician V. Lazaryan, Department of Electrical \\ Engineering and Electromechanics, Lazaryana Str. 2, room 238, 49000, Dnipro, Ukraine
}

\begin{abstract}
For the first time, the "field" approach for explaining the processes of transmission and generation of electric power losses in devices of electric transport systems is described and theoretically substantiated on the basis of the theory of electromagnetic field. The results of the solution of the system of electromagnetic field equations show that it is energetically appropriate to design low-floor types of electric rolling stock. A qualitative view of electric power flows arriving through the air of the feeder zone from the traction substation and entering to the electric rolling stock through the roof and the front part of its body is presented. It is established that the main flow of energy enters through the roof porcelain insulator. At the same time, the electromagnetic waves partly penetrate into the metal surfaces of roof and frontal part of the body, and partially they are reflected from them creating losses of active power. The results of calculations of these losses, power factor and reactive power factor of the electric locomotive roof are shown. The relation between the standing waves, formed in the feeder zone, and the reactive power consumed by the electric rolling stock is established.
\end{abstract}

\section{Introduction}

Existing scientific publications, highlighting findings of electric power processes in electric transport systems, are based on the "circuit approach", i.e. on the theory of linear or nonlinear electric and magnetic circuits. Though, in essence, all electromagnetic and electric power processes at their full and detailed studying require the "field approach" based on the theory of electromagnetic field. In our opinion, it is nearly the main reason why a set of processes or existing facts in electric power engineering is not explained sufficiently. This "circuit approach" restricts and limits possibilities of scientific management of power processes. In this respect a lot of things are unexplainable. For instance, in case of "leak" (i.e. losses of the electric power in electric traction system) the meter registrations of electric power of traction substations (TS) and electric rolling stock (ERS) differ. These losses are already called "conditional losses" and they are in a directive way regulated by the Main Department of locomotive facilities of Ukrzaliznytsia (Ukrainian Railway). And though, many authors are trying to justify the reasons of occurring and change of these losses, but in our opinion these arguments are not convincing.

And, after all, we present the most important justification of the above. According to the theoretical bases of electrical engineering, electromagnetic energy (electric power) is transmitted from the source (traction substation) to the consumer (electric locomotive) not trough the wires (i.e. electric circuit), but through the dielectric (air) between (and behind) the wires and it is transmitted not by charges, but by electromagnetic field, i.e. by electromagnetic waves.

In this regard, the author of the present work covers a set of electrical power issues concerning power transmission in electric transport systems. For this purpose we will recall that, as it is well-known from the theoretical electrical engineering, the power transmission (and information as well) through dielectric is characterized by the Poynting vector $\vec{S}$ in respect of both quantity and direction [1, p. 421], [2, p. 147], [3, p. 220]:

$$
\vec{S}=[\vec{E} \cdot \vec{H}],
$$

where $\vec{E}$ and $\vec{H}$ are vectors of electric and magnetic fields' intensities in dielectric points, i.e. the air in the feeder zone. The direction of the vector $\vec{S}$, i.e. direction of power flow in the given point, coincides with the forward movement of the right handed propeller at the rotation of vectors of $\vec{E}-\vec{H}$ from vector $\vec{E}$ to vector $\vec{H}$ towards a smaller corner between them.

\section{Elements of the theory of wave reflection and refraction}

As it is known from [1, pp. 590-601], [4, p. 363] if a plane electromagnetic wave, which varies sinusoidally in medium 1 with parameters $\varepsilon_{\mathrm{al}}, \mu_{\mathrm{al}}, \gamma_{\mathrm{al}}$, meets a surface of another medium 2 with parameters $\varepsilon_{\mathrm{a} 2}, \mu_{\mathrm{a} 2}, \gamma_{\mathrm{a} 2}$, and

\footnotetext{
* Corresponding author: nkostin@ukr.net
} 
this wave falls (incident wave with intensities $\vec{E}_{\text {in }}, \overrightarrow{\vec{H}}_{\text {in }}$ ) perpendicular to the border of mediums, then it is partially reflected $\vec{E}_{\mathrm{rf}}, \quad \overrightarrow{\underline{H}}_{\mathrm{rf}}$ with the reflection coefficient equals

$$
\underline{\rho}_{\mathrm{rf}}=\frac{\underline{Z}_{2}-\underline{Z}_{1}}{\underline{Z}_{2}+\underline{Z}_{1}}
$$

and can be described as

$$
\begin{aligned}
& \overrightarrow{\vec{E}}_{\mathrm{rf}}=\underline{\rho}_{\mathrm{rf}} \cdot \underline{\vec{E}}_{\mathrm{in}}, \\
& \underline{\vec{H}}_{\mathrm{rf}}=-\rho_{\mathrm{rf}} \cdot \underline{\vec{H}}_{\mathrm{in}} .
\end{aligned}
$$

Furthermore, this wave partially penetrates (refracts) $\vec{E}_{\mathrm{pn}}, \vec{H}_{\mathrm{pn}}$ into another medium 2 with the penetration coefficient (refraction) $\underline{\rho}_{\mathrm{pn}}$, which are defined for:

1) waves of electric field intensity according to the expressions:

$$
\begin{aligned}
& \underline{\rho}_{\mathrm{pn}}^{E}=\frac{2 \underline{Z}_{2}}{\underline{Z}_{2}+\underline{Z}_{1}}, \\
& \underline{\vec{E}}_{\mathrm{pn}}=\underline{\rho}_{\mathrm{pn}}^{E} \cdot \underline{\vec{E}}_{\mathrm{pn}} ;
\end{aligned}
$$

2) waves of magnetic field intensity according to the expressions:

$$
\begin{aligned}
& \underline{\rho}_{\mathrm{pn}}^{H}=\frac{2 \underline{Z}_{1}}{\underline{Z}_{2}+\underline{Z}_{1}}, \\
& \underline{\vec{H}}_{\mathrm{pn}}=\underline{\rho}_{\mathrm{pn}}^{H} \cdot \underline{\vec{H}}_{\mathrm{in}},
\end{aligned}
$$

where $\underline{Z}_{1}$ and $\underline{Z}_{2}$ are normally complex wave impedances of mediums 1 and 2. For dielectric medium, the impedances have to be defined as

$$
\underline{Z}_{\text {diel }}=\sqrt{\frac{\mu_{\mathrm{a}}}{\varepsilon_{\mathrm{a}}}},
$$

and they are real values. For a conducting medium, the impedances take imaginary value and should be defined according to the expression:

$$
\underline{Z}_{\text {cond }}=\sqrt{\frac{\omega \mu_{\mathrm{a}}}{\gamma}} e^{j 45^{0}},
$$

where $\omega=2 \pi f$ is an angular frequency of sinusoidally varying voltage and current which produce electromagnetic field (waves).

From (2), (9) and (10) it follows that if mediums 1 and 2 have identical parameters, the reflected wave is absent. In this case, the only incident wave is in medium 1 and refracted wave takes place in medium 2.

Irrespective of the incidence angle of the incident wave on the bounder of mediums 1 and 2, the refracted wave propagates in the conducting medium, first, always in the direction of a normal to the border of mediums and, secondly, this wave dies out at the depth $\delta$ defined as:

$$
\delta=\sqrt{\frac{2}{\omega \gamma_{2} \mu_{\mathrm{a} 2}}} .
$$

And finally the last remark: since waves, being reflected, don't change axes of vectors but can change their sign, instead of the corresponding vectors $\underline{\vec{E}}$ and $\underline{\vec{H}}$ it is possible to consider their vector modules.

\section{Power flow in the air between a catenary system and a rail}

Let's consider power transmission from a DC traction substation to an electric locomotive for the case of onesided supply and the most widespread catenary system of 2MF-100+A185 type and the rail of R65 type (Fig. 1)

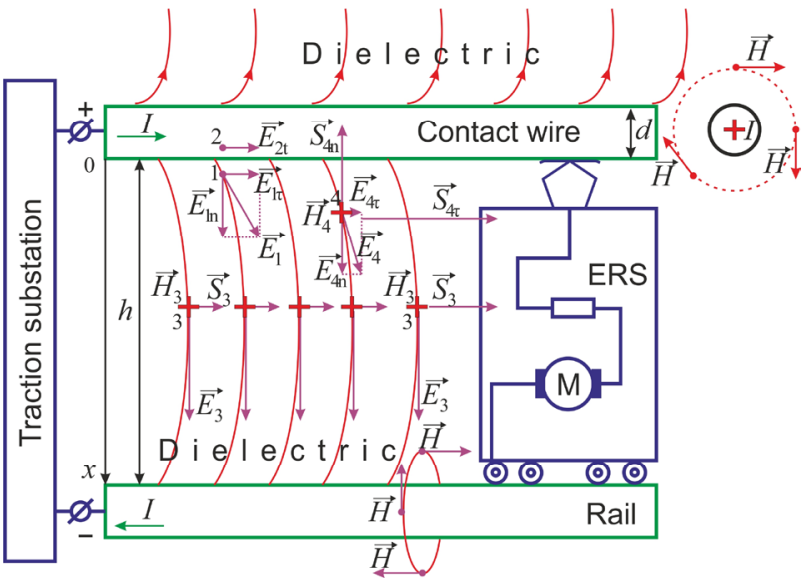

Fig. 1. A qualitative view of electricity transmission in the feeder zone.

The electromagnetic field in the dielectric (air) surrounding the catenary system is described by the system of equations [3, pp. 214-215], [5, pp. 29-30]:

$$
\begin{gathered}
\operatorname{rot} \vec{H}=0, \\
\operatorname{rot} \vec{E}=0, \\
\operatorname{div} \vec{B}=0, \\
\operatorname{div} \vec{D}=\operatorname{div} \varepsilon_{\mathrm{a}} \vec{E}=0 \\
\vec{B}=\mu_{\mathrm{a}} \vec{H}, \\
\vec{D}=\varepsilon_{\mathrm{a}} \vec{E}, \\
\vec{E}=-\operatorname{grad} V .
\end{gathered}
$$

Expression (13) demonstrates that electric field in the air is eddy-free, i.e. potential; therefore (18) is correct. Accepting that dielectric medium is homogenous, i.e. $\varepsilon_{\mathrm{a}}=$ const , and after inserting (18) in (15), we receive Laplace's equation

$$
\operatorname{divgrad} V=\frac{\partial^{2} V}{\partial x^{2}}+\frac{\partial^{2} V}{\partial y^{2}}+\frac{\partial^{2} V}{\partial z^{2}}=0,
$$

where the potential $V(x, y, z)$ is subjected in each dielectric point of the traction power system. Let's consider that in the first approximation the potential $V$ is changing only relatively to the coordinate $x$, i.e. from the catenary system to the rail (Fig. 1), then (19) should to be simplified:

$$
\frac{d^{2} V(x)}{d x^{2}}=0 .
$$

The solution of (20) is possible under desired boundary conditions [5, pp. 37-39] and one of them is the equality of tangential components of electric field intensity (Fig. 1): $\vec{E}_{1 t}=\vec{E}_{2 t}$. The component $\vec{E}_{2 t}$ in the conductor can be defined from well-known equation: 


$$
E_{2 t}=\frac{J}{\gamma},
$$

where $J$ is a current density in the catenary system, $\gamma$ is electrical conductivity of material of the system.

For example, feeder current at one train, operated by the DE1 electric locomotive, doesn't exceed $I=2000$ [A], and a cross section of the equivalent catenary system of $2 \mathrm{MF}-100+\mathrm{A} 185$ type has to be found according to $[6$, p. 52$]$ as:

$$
s_{\mathrm{eq}}=2 \cdot 100+\frac{185}{1.7}=308.82\left[\mathrm{~mm}^{2}\right]=308.82 \cdot 10^{-6}\left[\mathrm{~m}^{2}\right] \text {. }
$$

Hence,

$$
J=\frac{I}{s_{\text {eq }}}=\frac{2000}{308.82 \cdot 10^{-6}}=6.48 \cdot 10^{6}\left[\mathrm{~A} / \mathrm{m}^{2}\right] .
$$

If $\gamma$ for cooper equals $5.8 \cdot 10^{7}[\mathrm{~cm} / \mathrm{m}]$, then

$$
E_{2 t}=\frac{6.48 \cdot 10^{6}}{5.8 \cdot 10^{7}}=0.112[\mathrm{~V} / \mathrm{m}]
$$

At the same time a normal intensity component $\vec{E}_{1 \mathrm{n}}$ in dielectric is $E_{1 \mathrm{n}}>\frac{U}{h}$, since the field in the dielectric is non-homogenous and the strongest one is near catenary wires. Accepting maximal value of $h$, the intensity is calculated as

$$
E_{1 \mathrm{n}}>\frac{3300}{6,8}=485.3[\mathrm{~V} / \mathrm{m}]
$$

Hence, $E_{1 t}$ equals $E_{2 t}$ and is $0.112[\mathrm{~V} / \mathrm{m}]$, that is less than $E_{1 \mathrm{n}}$ in $4.3 \cdot 10^{3}$ times. Therefore, calculating the electric field in the air around the catenary system it is possible to ignore component $\vec{E}_{1 t}$ and use boundary electrostatics condition, i.e. condition of equipotential surface of a conductor. Hence, the intensity in the air between the catenary system and the rail is defined as

$$
E(x)=-\operatorname{grad} V(x)=-\frac{d V}{d x}=\frac{U}{h} .
$$

The intensity of magnetic field will be found using the Ampere's law (Fig. 1) as $H \cdot 2 \pi r_{0}=I$. Taking into account that $r_{0}=x+d / 2$, we get

$$
H(x)=\frac{I}{2 \pi(x+d / 2)},
$$

where $d$ is a diameter of the equivalent catenary wire.

At a further analysis we will take into account that to the strength $H(x)$, determined by (24) and produced by equivalent catenary wire, it is necessary to add the component produced by the current in rails. The equations for resultant intensity may to be found at any point (in two-wire power line), for example, according to [3, pp. 176-181]. Substitute (23) and (24) into (1), in the first approximation we get (without considering $\vec{H}$, produced by the current in a rail) the equation of the absolute value of the Poynting vector $\vec{S}$ in dielectric (air) points between the catenary system and the rail:

$$
|S|=\frac{U \cdot I}{2 \pi \cdot h(x+d / 2)} .
$$

In points $1,2,3$ and 4 (Fig. 1) the vector $\vec{H}$ is directed behind a flat surface of the sheet as shown in Fig. 1. Then, $\vec{E}_{4}$ is divided into two components: tangential component $\vec{E}_{4 x}$ and the normal $\vec{E}_{4 n}$ to the surface of the contact wire. Taking into account the above-mentioned direction rule of the Poynting vector $\vec{S}$, it is possible to make the following conclusions.

1) In point 3 , i.e. in the middle part of the space of traction network the vector $\vec{S}_{3}$, i.e. the energy flow, is completely directed from TS to electric locomotive.

2) In point 4 the component $\vec{E}_{4 n}$ and the vector $\vec{H}_{4}$, according to (1), cause the formation of component of Poynting vector $\vec{S}_{4 \tau}$ which also has the direction to the load (i.e. ERS).

3) In point 4 the components $\vec{E}_{4 \tau}$ and $\vec{H}_{4}$ also form the normal component $\vec{S}_{4 n}$ directed out of the dielectric (air) into the contact wire. On the border with the wire the electromagnetic wave falling from the air $\left(\vec{E}_{4 \tau}, \vec{H}_{4}\right)$ is partially reflected from the wire surface, and partially penetrates (refracts) into it; let's define the reflected wave.

For the air of medium 1, the wave impedance can be calculated as

$$
Z_{1}=\sqrt{\frac{\mu_{0}}{\varepsilon_{0}}}=\sqrt{\frac{4 \pi \cdot 10^{-7}}{8.854 \cdot 10^{-12}}}=376.7[\mathrm{Ohm}] .
$$

Medium 2 is the conducting medium and consist of wire of the $2 \mathrm{MF}-100+\mathrm{A} 185$ catenary system. The wire cross-section is equal to $308.82 \cdot 10^{-6}\left[\mathrm{~m}^{2}\right]$. At the same time the wire diameter is $19.83[\mathrm{~mm}]$, specific conductivity is $\gamma_{2}=\gamma_{\mathrm{eq}}=5.0 \cdot 10^{7}[\mathrm{Sm} / \mathrm{m}]$ and $\mu_{\mathrm{r} 2}=1.0$. Feeder current is inverted, 6- or 12-pulsing; and harmonics with the frequency of $300 \div 2400[\mathrm{~Hz}]$ are dominant in it. Then wave impedance of the material of the equivalent wire, in accordance with (10), is equal to:

$$
\underline{Z}_{2}=\sqrt{\frac{2 \pi \cdot 2000 \cdot 1 \cdot 4 \pi \cdot 10^{-7}}{5 \cdot 10^{7}}}=177.6 \cdot 10^{-7} \cdot \mathrm{e}^{j 45^{o}}[\mathrm{Ohm}] \text {. }
$$

Let's define the reflection coefficient on the border "air - wire surface" according to (2):

$$
\underline{\rho}_{\mathrm{rf} 12}=\frac{177.6 \cdot 10^{-7} \cdot e^{j 45^{0}}-376.7}{177.6 \cdot 10^{-7} \cdot e^{j 45^{0}}+376.7} \approx-1 .
$$

Then, in accordance with (3) and (4), field intensities of the reflected wave are $\underline{\vec{E}}_{\mathrm{rf} 1} \approx-\underline{\vec{E}}_{\mathrm{in} 1}$ and $\underline{\vec{H}}_{\mathrm{rf} 1} \approx \underline{\vec{H}}_{\mathrm{in} 1}$.

Hence, the reflected wave almost completely reflecting, changes the sign at the vector $\vec{E}$. Refraction coefficients according to (5) and (7) are:

$$
\begin{gathered}
\underline{\rho}_{\mathrm{pn} 2}^{E}=\frac{2 \cdot 177.6 \cdot 10^{-7} \cdot e^{j 45^{0}}}{177.6 \cdot 10^{-7} \cdot e^{j 45^{0}}+376.7} \approx 0.94 \cdot 10^{-7} e^{j 45^{0}} \\
\underline{\rho}_{\mathrm{pn} 2}^{H}=\frac{2 \cdot 376.7}{177.6 \cdot 10^{-7} \cdot e^{j 45^{0}}+376.7} \approx 20 .
\end{gathered}
$$


Despite the obtained values of $\rho_{\mathrm{pn}}$, a part of the incident wave is refracted. According to (11) it travels into the wire to the depth $\delta$ of:

$4.11[\mathrm{~mm}]$ for sinusoidal wave with frequency of $300[\mathrm{~Hz}]$; 1.45 [mm] for sinusoidal wave with frequency of 2400 [Hz].

Thus, the equivalent wire of the diameter $d=19.83[\mathrm{~mm}]$ of the catenary system is "impenetrable" for a wave; it dies out at the depth of $1.45 \div 4.11[\mathrm{~mm}]$. Energy of this wave is spent on the thermal losses in the wire occurring at feeder current flow in it. For comparison, let us calculate these losses in a kilometre of equivalent contact wire using existing (i.e. by the circuit theory) and proposed here "field" approach.

3.1) The circuit approach. For resistivity of contact wire $R_{\mathrm{cw}}=42.4 \cdot 10^{-3}[\mathrm{Ohm} / \mathrm{km}]$ and feeder current $I=2000[\mathrm{~A}]$ the losses according to the circuit approach are $\Delta P_{\mathrm{CA}}=R_{\mathrm{cw}} I^{2}=169.6[\mathrm{~kW} / \mathrm{km}]$.

3.2) The field approach. In this case, the losses are determined by the value of Poynting vector $\vec{S}$ at the surface of the contact wire (Fig. 1) as

$$
\Delta P_{\mathrm{FA}}=S_{s}=E_{1 t} \cdot H_{1} \cdot \sin \left(\vec{E}_{1 t}^{\wedge} \vec{H}_{1}\right) .
$$

The intensity $H_{1}$ has to be found according to the total current law as

$$
H_{1}=\frac{I}{2 \pi d / 2}=\frac{2000 \cdot 2}{2 \pi \cdot 19.83 \cdot 10^{-3}}=32120[\mathrm{~A} / \mathrm{m}],
$$

where $d$ is a diameter of equivalent contact wire. Taking into account calculated above $E_{1 t}=0.112[\mathrm{~V} / \mathrm{m}]$, value of Poynting vector equals

$$
S_{s}=0.112 \cdot 32120 \cdot \sin 90^{\circ}=3597.44\left[\mathrm{~W} / \mathrm{m}^{2}\right] \text {. }
$$

The surface of a kilometre of equivalent contact wire is $62.266\left[\mathrm{~m}^{2}\right]$, so finally the losses according to the field approach are $\Delta P_{\mathrm{FA}}=225.4[\mathrm{~kW} / \mathrm{km}]$.

As follows from the calculation results, $\Delta P_{\mathrm{FA}}$ exceeds $\Delta P_{\mathrm{CA}}$ by $24.7 \%$, which corresponds to so-called "conditional" losses, "leakage" losses, which are still inexplicable and which are not counted by electricity meters, since their principle of operation is based on the circuit theory. Therefore, the electric power analysis of electric transport systems have be carried out on the basis of the electromagnetic field theory.

4) According to (25), the highest power density per time is transmitted in the space of small values $x$ (Fig. 1), that is, in the space around the contact wires and the rail.

It is possible to show that the same condition regarding the direction of electric power is observed also in the space above the catenary system and under the rail base with the ground; but values of the vector $\vec{S}$ will be different, considering non-homogeneity of all traction network.

Considering the above and expression (25), it is possible to make the following strategic proposals regarding devices of electric traction system. According to (25), with the decrease of the value $h$, i.e. with the decrease of distance between the contact wire and the head of a rail, density of the electric power transmitted from TS to ERS increases. According to the technical safety rules, $h_{\max }=6.8[\mathrm{~m}]$ and $h_{\min }=5.75[\mathrm{~m}]$, therefore in the projected electric traction systems it is reasonable to reduce $h$, and construct low-floor (but probably longish) types of ERS. There is nothing irregular in this proposal if we take into account that nowadays one of the most important indicators of technical progress is the reducing the space occupied by the electromagnetic device $[7,8]$, as it is for a reason microcomputers, electrical micro machines, different micro devices and the like are created. In the context of energetics for production of the mentioned devices it is reasonable to enter the coefficient of utilization of construction space $\eta_{\mathrm{e}}$ :

$$
\eta_{\mathrm{e}}=S_{\min } / S_{\max }
$$

where $0 \leq \eta_{\mathrm{e}} \leq 1$.

\section{Electric power flow through the roof bushing insulator and through the roof of electric locomotive}

Out of the total electric power, flowing (carried by waves) to ERS out of the air space of the traction network it is possible to identify two flows: the main and the minor. The main flow is the energy flow representing the greatest part of the energy received by ERS. It concentrates around traction network wires and enters to electrical devices of the high-voltage cabinet through the roof bushing insulator, i.e. roof surface (Fig. 2) and through the bottom of the cabinet from the space around the rail. The minor flow is the electric power flow carried by waves which fall on the front part of electric locomotive body, which consists, in its turn, of metal and dielectric (cab windscreen) parts (Fig. 2).

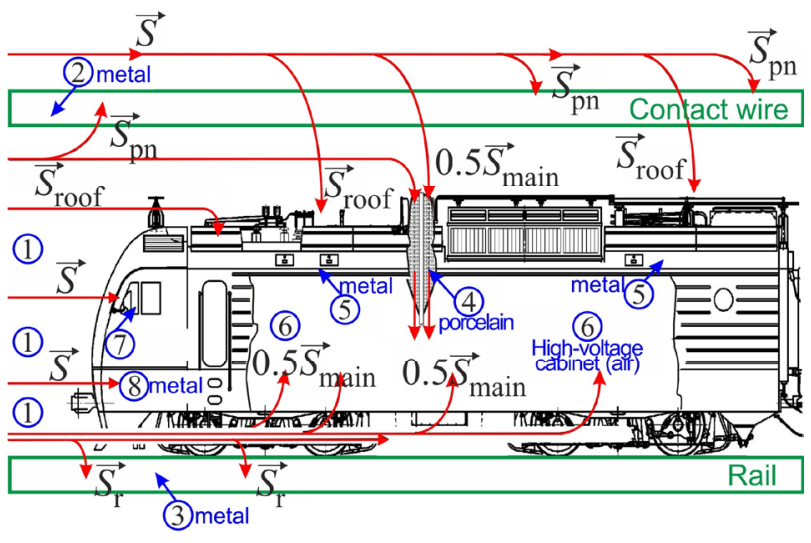

Fig. 2. A qualitative view of the flows of electricity through the roof and the frontal part of ERS: 1 - air around wires and rails of traction network; 2 - contact wire; 3 - rail; 4 - insulator of roof bushing; 5 - metal of the roof; 6 - air in the high-voltage cabinet; 7 - cab windscreen; 8 - metal part.

In the beginning we will analyse the main flow in which it is possible to identify the following components of the Poynting vector carrying it (Fig. 2). 
1) The main vector $\left(0.5 \cdot \vec{S}_{\text {main }}\right)$ corresponding to the energy flow density coming into the elements of the traction power circuit of ERS (into the high-voltage cabinet) through the roof bushing porcelain insulator. In order to analyse the electromagnetic waves carrying $\vec{S}_{\text {main }}$ we will define the coefficients of reflection $\underline{\rho}_{\mathrm{r} 14}$ and refraction $\underline{\rho}_{\text {pn14 }}$ on the border "air 1 - porcelain 4 ". For ideal dielectrics like air $\left(\varepsilon_{\mathrm{r} 1}=1.0\right)$ and porcelain $\left(\varepsilon_{\mathrm{r} 4}=6.0\right)$, expressions (2) and (5) for $\underline{\rho}_{\mathrm{rf}}$ and $\underline{\rho}_{\mathrm{pn}}$ are simplified as:

$$
\begin{gathered}
\rho_{\mathrm{r} 14}=\frac{\sqrt{\varepsilon_{\mathrm{r} 1}}-\sqrt{\varepsilon_{\mathrm{r} 4}}}{\sqrt{\varepsilon_{\mathrm{r} 1}}+\sqrt{\varepsilon_{\mathrm{r} 4}}}=\frac{\sqrt{1}-\sqrt{6}}{\sqrt{1}+\sqrt{6}}=-0.42, \\
\rho_{\mathrm{pn} 4}^{E}=\frac{2 \sqrt{\varepsilon_{\mathrm{r} 1}}}{\sqrt{\varepsilon_{\mathrm{r} 1}}+\sqrt{\varepsilon_{\mathrm{r} 4}}}=\frac{2 \cdot \sqrt{1}}{\sqrt{1}+\sqrt{6}}=0.58 .
\end{gathered}
$$

Therefore:

$$
\begin{gathered}
E_{\mathrm{r} 14}=-0.42 E_{\mathrm{in} 1}, \\
\rho_{\mathrm{pn} 4}^{H}=\frac{2 \sqrt{\varepsilon_{\mathrm{r} 4}}}{\sqrt{\varepsilon_{\mathrm{r} 1}}+\sqrt{\varepsilon_{\mathrm{r} 4}}}=1.42, \\
H_{\mathrm{r} 14}=-\left(-0.42 H_{\mathrm{in} 1}\right)=0.42 H_{\mathrm{in} 1}, \\
E_{\mathrm{pn} 4}=0.58 E_{\mathrm{in} 1}, \\
H_{\mathrm{pn} 4}=1.42 H_{\mathrm{in} 1} .
\end{gathered}
$$

As we can see, a part of the energy is reflected from the insulator. To minimise this part, it is necessary to select this insulator with $\varepsilon_{\mathrm{r}}$, close to $\varepsilon_{\mathrm{r}}=1.0$.

The second half of $\vec{S}_{\text {main }}$ (Fig. 2) enters into the highvoltage cabinet of ERS from the space around the rail (i.e. "from the bottom" of ERS). Wave propagation in this space and power transmission needs more in-depth studying which is not given here yet.

2. The power vector (Poynting) $\vec{S}_{\text {roof }}$ penetrates into the metal roof of ERS through its surface (Fig. 2). The vector $\vec{S}_{\text {roof }}$ is directed normally to the surface. Roof material is a sheet structural steel of $2[\mathrm{~mm}]$ thick with parameters: $\gamma=7 \cdot 10^{6}[\mathrm{Sm} / \mathrm{m}] ; \mu_{\mathrm{r}}=1000$.

Electromagnetic harmonic waves of various frequencies, falling from air 1 onto the border with the surface of metal roof 5 (Fig. 2), are partially reflected, and partially penetrate into the metal of the roof, gradually dying out and at the same time causing losses of electric power in roof metal.

Wave impedance of the roof metal $\underline{Z}_{5}$, found according to (10) for $f=2400[\mathrm{~Hz}]$, equals $164.4 \cdot 10-5 \mathrm{e}^{j 45^{0}}[\mathrm{Ohm}]$. For air it equals to $-\underline{Z}_{1}=376.7[\mathrm{Ohm}]$. Substituting $\underline{Z}_{1}$ and $\underline{Z}_{5}$ in (2), the coefficient of wave reflection from the roof surface is $\rho_{\text {rf } 15} \approx-1$. Substituting them in (5) the refraction coefficient equals $\rho_{\text {pn15 }} \approx 0.87 \cdot 10^{-5} e^{j 45^{0}}$. The wave with the frequency of $2400[\mathrm{~Hz}]$ penetrates on the depth $\delta_{5}$ of $0.128[\mathrm{~mm}]$, analogically, with frequency of $600[\mathrm{~Hz}]-$ $0.246[\mathrm{~mm}]$, and with frequency of $50[\mathrm{~Hz}]-0.85[\mathrm{~mm}]$.
As far as, the thickness of the steel roof sheet is 2 [mm] and $\delta_{5 \max }=0.85[\mathrm{~mm}]$, the metal of the roof is "impenetrable" for the waves since the wave dies out completely, without reaching an opposite surface. It means that the metal sheet of the roof can be considered as an endlessly thick sheet or as endless conducting halfspace, and the power dissipation in it is equal to the Poynting vector on the surface of the roof $\vec{S}_{\text {roof }}$ multiplied by this surface $s_{\text {roof }}$.

Complex Poynting vector on the surface of the sheet is defined by the expression [8]:

$$
\underline{S}_{S}=(1+j) \sqrt{\frac{\omega \mu_{\mathrm{a}}}{2 \gamma}} \cdot \frac{H_{m S}^{2}}{2} .
$$

Its active component is

$$
S_{P}=\alpha_{P} \sqrt{\frac{\omega \mu_{\mathrm{a}}}{2 \gamma}} \cdot \frac{H_{m s}^{2}}{2},
$$

and inductive component is

$$
S_{Q}=\alpha_{Q} \sqrt{\frac{\omega \mu_{\mathrm{a}}}{2 \gamma}} \cdot \frac{H_{m S}^{2}}{2},
$$

where for steel $\alpha_{\underline{P}}=1.4, \alpha_{Q}=0.85, H_{m S}$ is an amplitude of harmonic magnetic field intensity on the given surface.

Let's calculate $H_{m S}$ for a harmonic with frequency of $f=600[\mathrm{~Hz}]$ (for a 12-pulse current). The value of $H_{m S}$ for this harmonic is $955.5[\mathrm{~A} / \mathrm{m}]$. Then, applying necessary values and parameters of roof steel sheet in (28) and (29), we get:

- losses of active power from the harmonic per roof surface unit

$$
S_{P}=371.6\left[\mathrm{~W} / \mathrm{m}^{2}\right] ;
$$

- consumption of reactive power

$$
S_{Q}=225.6\left[\mathrm{var} / \mathrm{m}^{2}\right] .
$$

Total surface of the roof of one ERS section equals $50\left[\mathrm{~m}^{2}\right]$, let $s_{\text {roof }} \approx 100\left[\mathrm{~m}^{2}\right]$ for a "medium-active" surface of two-section ERS. Then the power losses are

$$
\begin{aligned}
& P=37.2[\mathrm{~kW}], \\
& Q=22.6[\mathrm{kvar}], \\
& S=43.6[\mathrm{kVA}] .
\end{aligned}
$$

Power factor of the roof equals $\lambda=0.85$ and reactive power factor is $\operatorname{tg} \phi=0.61$.

\section{Energy flow falling on the front of the locomotive body}

Electromagnetic waves propagating in the middle part of the airspace 1 between the catenary system and the rail, fall onto the front part of the locomotive body which consists of glass 7 and lower metal part 8 (Fig. 2). The thickness of cab windscreen is $15 \mathrm{~mm}$ and $\varepsilon_{\mathrm{r} 7}=5.5 \div 10$ (let's take 9.0). On the border "air $1-$ glass 7" waves are partially reflected with the coefficient of 


$$
\rho_{\mathrm{r} 17}=\frac{\sqrt{\varepsilon_{\mathrm{r} 1}}-\sqrt{\varepsilon_{\mathrm{r} 7}}}{\sqrt{\varepsilon_{\mathrm{r} 1}}+\sqrt{\varepsilon_{\mathrm{r} 7}}}=\frac{\sqrt{1}-\sqrt{9}}{\sqrt{1}+\sqrt{9}}=-0.5,
$$

and partially penetrated (refracted) with the coefficient of

$$
\rho_{\mathrm{pn} 7}^{E}=\frac{2 \sqrt{\varepsilon_{\mathrm{r} 1}}}{\sqrt{\varepsilon_{\mathrm{r} 1}}+\sqrt{\varepsilon_{\mathrm{r} 7}}}=\frac{2 \sqrt{1}}{\sqrt{1}+\sqrt{9}}=0.5 .
$$

Then, $E_{\mathrm{rf} 1}=-0.5 E_{\mathrm{in} 17}$ and $E_{\mathrm{pn} 7}=-0.5 E_{\mathrm{in} 17}$.

The metal part of lower front part of ERS body is made from structural sheet steel of $7 \mathrm{~mm}$. And therefore, analogically to the roof metal, it is also "impenetrable" for electromagnetic waves, so a part of the wave, penetrating into metal, creates the power losses [9] determined by $(27) \div(29)$. In order to find them we must know amplitudes of harmonicas of the magnetic intensity $H_{m S}$ on the surface of metal part of the body which are not given here yet.

\section{Relationship between reactive-power and standing electromagnetic waves}

In addition to the considered above, let's analyse a variable electromagnetic field in the air space of feeder zone. This field is created by the harmonic flat progressing waves of electric and magnetic field intensities that transmit electric power from the TS to the electric locomotive [5]:

$$
\begin{aligned}
& E(y, t)=E_{m \text { in }} \sin (\omega t-\beta y)+E_{m \text { rf }} \sin (\omega t+\beta y), \\
& H(y, t)=H_{m \text { in }} \sin (\omega t-\beta y)+H_{m \text { rf }} \sin (\omega t+\beta y),
\end{aligned}
$$

where: $\quad \beta=\omega / C_{0} \quad$ is a phase coefficient; $C_{0} \approx 3 \cdot 10^{8}[\mathrm{~m} / \mathrm{s}]$. In expressions (30) and (31) the first components of electric and magnetic fields describes the incident (falling) wave, and the second - reflected (reverse) electromagnetic waves.

It is shown above that on the border with the metallic surfaces of the contact wire, roof and front part of the ERS body the wave reflection coefficient is practically equal to 1 , i.e. incident wave is reflected practically fully. In this case the amplitudes of incident (falling) and reflected waves are identical and this influences on appearing of standing waves.

The propagating harmonic wave with the frequency of $50[\mathrm{~Hz}]$ has length of $\lambda=6000[\mathrm{~km}]$ in the air. So, the standing wave on distance between nodes of $\lambda / 4=1500[\mathrm{~km}]$ completely fills a feeder zone. Then, in the running out or idle modes of ERS's traction transformer, or in the regenerative braking mode, when there are no any consumers of recovered power in the section or TS, the active power is not transmitted on a feeder zone. At the same time, on every area which is $\lambda / 4$ long (i.e. on all feeder zone) there is an oscillating process of power exchange of electric and magnetic fields $\left(\frac{\mu_{\mathrm{a}} H^{2}}{2} \leftrightarrow \frac{\varepsilon_{\mathrm{a}} E^{2}}{2}\right)$, existing in the zone. An electromagnetic wave at this time in a feeder zone "stands", does not move and does not transfer energy from TS to ERS. Consequently, all time-varying energy of the electromagnetic field, according to (9) and (10), is reactive $Q$. Thus, in order to compensate $Q$ it is necessary to suppress the standing wave in a feeder zone. One of the ways is to place a medium with a certain electromagnetic properties in front of ERS. The second method is to cover the surfaces (of contact wire and ERS body) with a substance having dielectric capacitance $\varepsilon_{\mathrm{r} 2}$, close to $\varepsilon_{\mathrm{r} 1}$, i.e. air, because in this case, according to (2), $\rho_{\mathrm{rf}}$ is far from 1 , i.e. the reflected wave is practically missing.

\section{Conclusions}

1) The "field" approach, based on the theory of electromagnetic field, for the proving of transmission and formation of electric power losses in the devices of electric transport systems is more fundamental, and therefore more reliable than the existing "circuit" principles of such analysis based on the theory of electric circuits.

2) Flows of electromagnetic energy go from a traction substation to an electric locomotive through the air between a contact wire and a rail going into a highvoltage cabinet and then into traction engines through the roof porcelain insulator and metal parts of locomotive underbody part.

3) Electromagnetic waves falling from the dielectric (air) onto the surface of contact wire and metal part of the roof are partially reflected, and partially penetrate into the metal, gradually fading and at the same time creating energy losses in them.

4) The appearing and superposition of reflected and incident waves results in appearing of the standing waves in the air of the feeder zone, which create reactive power, as the last characterizes stationary waves. Therefore, in order to compensate reactive power it is necessary to suppress the mentioned waves.

\section{References}

1. A. Shadowitz, The Electromagnetic Field (Reprint by Dover Publications INC., NY 2010)

2. J. Turowski and M. Turowski, Engineering Electrodynamics: Electric Machine, Transformer and Power Equipment Design. (CRC Press Taylor \& Francis Group, NY 2014)

3. M. O. Kostin and O. H. Sheikina, Theory of Electrical Engineering, Vol. 3, Part 2 (Publ. Dep. of DNURT, Dnipropetrovsk 2011) [in Ukrainian]

4. D. M. Cook, The Theory of the Electromagnetic Field (Reprint by Dover Publications Inc., NY 2011)

5. V. Yu. Pimenov, V. I. Volman and A. D. Muravtsov, Technical Electrodynamics (Radio and connection, Moscow 2000) [in Russian]

6. K. G. Markvardt, Power supply of electrified railways (Transport, Moscow 1982) [in Russian] 